\title{
Chebyshev Expansions for Integrals of the Error Function
}

\section{By Van E. Wood}

1. Introduction. The repeated integrals of the error function [1, Chapter 7] are defined by

$$
\begin{aligned}
& i^{n} \operatorname{erfc} z=\int_{z}^{\infty} i^{n-1} \operatorname{erfc} t d t, \quad(n=0,1,2, \cdots), \\
& i^{0} \operatorname{erfc} z=\operatorname{erfc} z, \quad i^{-1} \operatorname{erfc} z=2 \pi^{-1 / 2} e^{-z^{2}} .
\end{aligned}
$$

From the recurrence relation

$$
i^{n} \operatorname{erfc} z=-z n^{-1} i^{n-2} \operatorname{erfc} z+(2 n)^{-1} i^{n-2} \operatorname{erfc} z, \quad(n=1,2,3, \cdots),
$$

the integrals may be calculated for small $z$, although with considerable loss of accuracy. For large $z$, backward recurrence may be used [2]; this is certainly the best method if one needs several of these functions for fairly large arguments, but if one wants values of a single function for a large range of arguments, it is very convenient to use Chebyshev expansions. In this note we present such expansions for the cases $n=1$ and $n=2, z$ real and nonnegative.

2. General Remarks. The integrals of the error function may be expressed in terms of generalized hypergeometric functions as follows:

$$
\begin{aligned}
i^{n} \operatorname{erfc} z= & 2^{-n} \sum_{k=0}^{n-2} \frac{(-2 z)^{k}}{k ! \Gamma\left(1+\frac{n-k}{2}\right)}+\frac{(-z)^{n}}{n !}+\frac{(-z)^{n-1}}{\pi^{1 / 2} \Gamma(n)} \\
& \times{ }_{2} F_{2}\left(-\frac{1}{2}, 1 ; \frac{n}{2}, \frac{n+1}{2} ;-z^{2}\right) \\
= & \frac{e^{-z^{2}}}{\pi^{1 / 2} 2^{n} z^{n+1}}{ }_{2} F_{0}\left(\frac{n+1}{2}, \frac{n+2}{2} ;-z^{-2}\right) .
\end{aligned}
$$

The first expression is closely related to the recurrence relation (2) and also suffers from cancellation of terms, but for the cases of interest here can be used for $z<1$, as explained further below. In the cases $n=1,2$, the ${ }_{2} F_{2}$ reduces to a confluent hypergeometric function. All we wish to do in this case is to give Chebyshev expansions for these hypergeometric functions, thus making the evaluation of the series a little more efficient. The expression (3b) is just the usual asymptotic expansion for the integrals of the error function [1], [3]; by expanding the ${ }_{2} F_{0}$ in Chebyshev polynomials, this asymptotic series is converted to a rapidly convergent, easily evaluated form, as discussed by Clenshaw [4]. The coefficients occurring in the expansions of the hypergeometric functions in terms of Chebyshev polynomials may be expressed in terms of generalized hypergeometric functions of higher order,

Received October 10, 1966. 
as discussed by Fields, Wimp, and Luke [5], [6], [7], but for numerical calculation of these coefficients it is somewhat easier in the present case to use the solution of the differential equation to obtain a recurrence relation for the coefficients [4]. The recurrence relations for the confluent functions are easily found f for the asymptotic expansion the appropriate differential equation is

$$
v^{3} f^{\prime \prime}+2\left(k^{2}+(n+2) v^{2}\right) f^{\prime}+(n+1)(n+2) f=0
$$

where

$$
\pi^{1 / 2} 2^{n} f={ }_{2} F_{0}\left(\frac{1}{2}(n+1), \frac{1}{2}(n+2) ;-z^{-2}\right)=\frac{1}{2} \sum_{r=0}^{\infty} \epsilon_{r} a_{2 r} T_{2 r}(v) ;
$$

$$
v=k z^{-1} ; \epsilon_{r}=2-\delta_{r_{0}} \text {. }
$$

The $a$ 's are then found to satisfy the relations

$$
\begin{aligned}
(r+n)(r+n-1) a_{r-2}= & (r-n)(r-n+1) a_{r+2}-2\left((2 k)^{2}+2 n+1\right) r a_{r} \\
& -2 r\left(a_{r-1}^{\prime}+a_{r+1}^{\prime}\right) ; \\
a_{r-1}^{\prime}= & a_{r+1}^{\prime}+2 r a_{r} ; \quad r=2,4,6, \ldots .
\end{aligned}
$$

3. Results and Discussion. We obtain for the first two integrals of the error function

(6a) $\pi^{1 / 2} i \operatorname{erfc} z=-\pi^{1 / 2} z+\frac{1}{2} \sum \epsilon_{r} b_{r} T_{2 r}(z)=\frac{1}{4} z^{-2} e^{-z^{2}} \sum \epsilon_{r} c_{r} T_{2 r}\left(z^{-1}\right) ;$

\begin{tabular}{|c|c|c|c|c|}
\hline$r$ & $b_{r}$ & $c_{r}$ & $d_{r}$ & $e_{r}$ \\
\hline 0 & 2.8929827 & 1.3618413 & 2.3109853 & 1.0388528 \\
\hline 1 & .4300235 & -.2409343 & .1519739 & -.3229885 \\
\hline 2 & -.0156956 & .0560098 & -. 34009 & .1028703 \\
\hline 3 & $\begin{array}{l}\text {. } 7391\end{array}$ & -.0152168 & 1139 & -.0347257 \\
\hline 4 & 319 & . 45926 & - & .0123637 \\
\hline 5 & . & 一. 14980 & . & -. 46072 \\
\hline 6 & & 5192 & & 17850 \\
\hline 7 & & 1890 & & 7152 \\
\hline 8 & & 717 & & 2951 \\
\hline 9 & & 282 & & 1249 \\
\hline 10 & & 114 & & 541 \\
\hline 11 & & - . & & 239 \\
\hline 12 & & 21 & & 108 \\
\hline 13 & & - . & & - . \\
\hline 14 & & 4 & & 23 \\
\hline 15 & & - & & - . \\
\hline 16 & & & & 5 \\
\hline 17 & & & & - \\
\hline 18 & & & & 1 \\
\hline
\end{tabular}

(6b) $4 i^{2} \operatorname{erfc} z=1+2 z^{2}-2 \pi^{-1 / 2} z \sum \epsilon_{r} d_{r} T_{2 r}(z)=\frac{1}{2} \pi^{-1 / 2} z^{-3} e^{-z^{2}} \sum \epsilon_{r} e_{r} T_{2 r}\left(z^{-1}\right)$;

where the coefficients $b, c, d, e$, are given to 7 decimal places in Table I. Using the expansions in $T_{2 r}(z)$ for $z<1$ and those in $T_{2 r}\left(z^{-1}\right)$ for $z>1$, one can calculate

TABLE I

Numerical values of expansion coefficients occurring in Eq. 6 
$i$ erfc $z$ and $i^{2}$ erfc $z$ correct to 6 significant figures ( 7 s.f. for $z>1$ ) using single precision on a computer with word length of 8 decimal places, for all $z$ for which $e^{-z^{2}}$ can be calculated correctly. To obtain greater accuracy, it is necessary either to use double precision or to use more than two different expansions for each function. From Gautschi's formula [2] for the number of terms required for calculation by backward recurrence, we see that that method will be better (for 7 s.f. accuracy) if all the $z$ 's of interest are greater than about 2.5. The advantage accruing from the use of Chebyshev approximations would be still greater for multiple-precision calculations of very high accuracy.

Battelle Memorial Institute

Columbus, Ohio

1. M. Abramowitz \& I. A. Stegun, (Editors), Handbook of Mathematical Functions, with Formulas, Graphs, and Mathematical Tables, National Bureau of Standards Applied Mathematics Series, No. 55, U. S. Government Printing Office, Washington, D. C., 1964; reprint, 1965. MR 29 \#4914; MR 31 \#1400.

2. W. GaUtSCHI, "Recursive computation of the repeated integrals of the error function," Math. Comp., v. 15, 1961, pp. 227-232. MR 24 \#B2113.

3. W. R. WILCOX "Asymptotic computation of the repeated integrals of the error function complement," Math. Comp., v. 18, 1964, pp. 98-105. MR $28 \# 1327$.

4. C. W. Clenshaw, "The numerical solution of linear differential equations in Chebyshev series," Proc. Cambridge Philos. Soc., v. 53, 1957, pp. 134-149. MR 18, 516.

5. J. L. FIELDS \& J. WIMP, "Expansions of hypergeometric functions in hypergeometric functions," Math. Comp., v. 15, 1961, pp. 390-395. MR 23 \#A3289.

6. J. WIMP \& Y. L. LUKE, "Expansion formulas for generalized hypergeometric functions," Rend. Circ. Mat. Palermo, 2, v. 11, 1962, pp. 351-366. MR 29 \#3681.

7. Y. L. LUKE \& J. WIMP, "Jacobi polynomial expansions of a generalized hypergeometric function over a semi-infinite ray," Math. Comp., v. 17, 1963, pp. 395-404. MR 28 \#255.

\section{An Integral Representation for the Modified Bessel Function of the Third Kind, Computable for Large, Imaginary Order}

\section{By James D. Lear and James E. Sturm}

The one-dimensional Schroedinger equation describing the quantum-mechanical motion of a particle of total energy $E$ and mass $\mu$ in a potential field of the form:

$$
\begin{array}{ll}
V=B \exp (-r / a) & \text { for } r>0 \\
V=\infty & \text { for } r \leq 0
\end{array}
$$

has, as time-independent solutions, the functions

$$
\left(\frac{\nu \sinh \pi \nu}{\pi}\right)^{1 / 2} K_{i \nu}(z)
$$

where $\nu=2 a\left(2 \mu E / \hbar^{2}\right)^{1 / 2}, z=2 a B e^{-r / 2 a}, K_{i \nu}(z)$ is the modified Bessel function of the third kind, and the normalization is to unit amplitude of the asymptotic ( $r$ increasing) solution [1]. In attempting to compute values for $K_{i \nu}(z)$ through use of the representation: 